\title{
KULTURELLE AKTEURE UND DIE DEUTSCH- FRANZÖSISCHE ZUSAMMENARBEIT: FORMEN, ZIELE, EINFLUSS
}

"Deutsch-französische Beziehungen entwickeln sich zunächst vor allem auf kulturellem und wirtschaftlichem Gebiet und im Bereich der Kontakte von Mensch zu Mensch, dann allmählich auch zunehmend zwischen den Regierungen, die sich aktiv um Verständigung bemühen «. ${ }^{1}$ Die in dieser Einschätzung eines bundesdeutschen Diplomaten vom Juli 1963 zum Ausdruck kommenden Interdependenzen zwischen den verschiedenen Ebenen der transnationalen Beziehungen zwischen der Bundesrepublik und Frankreich nach 1945/49 fanden lange keinen Eingang in die Historiographie, die sich über Jahrzehnte fast ausschließlich der politisch-diplomatischen Komponente dieses bilateralen Verhältnisses gewidmet und mit dieser Konzentration auf die gouvernementale Ebene »das Untersuchungsobjekt in ganz unzulässiger und unproduktiver Weise« verkürzt hatte. ${ }^{2}$ Obwohl sich seit einiger Zeit sowohl bei der Erforschung der internationalen Beziehungen im allgemeinen ${ }^{3}$ als auch für den Fall der deutsch-französischen Beziehungen immer stärker die Tendenz abzeichnet, die diplomatiegeschichtliche und gesellschafts- bzw. kulturgeschichtliche Ebene miteinander zu kombinieren, um die Interaktionsformen zwischen gouvermementalen und zivilgesellschaftlichen Akteuren in internationalen Entscheidungs- und Implementationsprozessen herauszuarbeiten, be-

1 Gedankenskizze für einen Vortrag über die Neuorientierung der politischen Beziehungen zwischen Frankreich und Deutschland nach 1945 [Juli 1963]; PAAA, B 24, Bd. 473, Bl. 205-220, hier Bl. 210.

2 Hans Manfred Bock, Wiederbeginn und Neuanfang in den deutsch-französischen Gesellschafts- und Kulturbeziehungen 1949 bis 1950, in: Lendemains, 21 (1996) 84, S. 58-66, hier S. 60.

Vgl. Wilfried LOTH u.a. (Hg.), Internationale Geschichte. Themen - Ergebnisse - Aussichten, München 2000; Ursula LEHMKUHL, Diplomatiegeschichte als internationale Kulturgeschichte: Theoretische Ansätze und empirische Forschung zwischen Historischer Kulturwissenschaft und Soziologischem Institutionalismus, in: Geschichte und Gesellschaft, 27 (2001) 3, S. 394-423. 
stehen bis heute zahlreiche Desiderata auf dem Feld der gesellschaftlichen und kulturellen Akteure. ${ }^{4}$

Ausdruck dieser Forschungslücken scheint es zu sein, daß die Funktionen und Aufgaben der kulturellen und gesellschaftlichen Akteure im (west-) deutsch-französischen Annäherungsprozeß weiterhin nicht eindeutig definiert sind. Während der ehemalige Bundesaußenminister Gerhard Schröder nach der Gründung des Deutsch-Französischen Jugendwerks (DFJW) bemerkte, $\mathrm{da} B$ die Idee zu seiner Schaffung 1962/63 win der Luft" gelegen habe, ${ }^{5}$ ohne jedoch zu erklären, durch wen die "Luftveränderungen " seit 1945 herbeigeführt worden seien, ist der Élysée-Vertrag für die junge Historikerin Gesa Bluhm ein Beispiel dafür, wie "politisches Vertrauen zwischen zwei Ländern fast aus dem Nichts heraus erfolgreich beschworen wird und die weitere $\mathrm{Zu}$ kunft beeinflussen konnte [...]. Vertrauen wurde, bevor es überhaupt ivon unten $\mathrm{zu}$ wachsen begonnen hatte, durch diesen Akt zur moralischen Ressource aufgebaut und verfestigt. ${ }^{6}$ Diese gegensätzlichen Positionen werfen die Frage auf, ob der 22. Januar 1963 auf halbem Wege oder noch am Anfang des Aussöhnungsprozesses stand, so wie verschiedene Festredner zum 40. Jahrestag des Élysée-Vertrages verlauten ließen.

So gilt es in diesem Beitrag nicht nur zu fragen, wann die Einsicht im Lernproze $\$ Oberhand gewann, daß das gegenseitige Mißtrauen zwischen beiden Nationen und die immer wieder aufflackernde Feindseligkeit "verderblich war, verderblich für sie selbst, für Europa, für die Welt«, wie es die bundesdeutsche Diplomatie formulierte. ${ }^{7}$ Über die Analyse der personellen, strukturellen und ideellen Verflechtungen zwischen den zivilgesellschaftlichen Kräften und den kulturpolitischen Akteuren in der Bundesrepublik und in Frankreich gilt es zudem Antworten zu suchen, über welche Zielvorstellungen, Praktiken und Verfahren sie Annäherungs- und Wandlungsprozesse zwischen der (west-)deutschen und französischen Gesellschaft anstießen und mitgestalteten.

4 Vgl. Hans Manfred BocK, Gesellschaftliche Neubegründung interkulturellen Austauschs. Zur Vorgeschichte und Struktur des Deutsch-Französischen Jugendwerks 1949-1963. Einleitung, in: Lendemains, 27 (2002) 107/108, S. 139-145.

5 Zit. nach Henri MÉNUDIER, Das Deutsch-Französische Jugendwerk. Ein exemplarischer Beitrag zur Einheit Europas, Bonn 1991, S. 66.

- Gesa BLUHM, Vertrauensarbeit. Deutsch-französische Beziehungen nach 1945, in: Ute FrEVERT (Hg.), Vertrauen. Historische Annähenung, Göttingen 2003, S. 365-393, hier S. 372, 377.

7 Note der Bundesregierung an die sowjetische Regierung, 29.3.1963; PAAA, B 24, Bd. 473, Bl. 154. 


\section{Transnationale Akteure zwischen Staat und Zivilgesellschaft}

\section{Die französische Kulturpolitik in Deutschland nach 1945 zwischen Säuberung und Umerziehung}

Nach drei Kriegen innerhalb von 80 Jahren und den gerade überwundenen années noires war es die "furchtbare Erinnerung" an die nationalsozialistischen Verbrechen, die eine kollektive Erfahrung, die das deutsch-französische Verhältnis im Jahre 1945 prägte. Auf die andere Erfahrung weist der französische Germanist Edmond Vermeil in seinem Vorwort zu Alfred Grossers Buch "L'Allemagne de l'Occident" hin, nämlich auf den Willen in der französischen Besatzungsverwaltung, die Konstellation, die die deutsche Niederlage mit sich gebracht hatte, für eine Normalisierung der Beziehungen mit dem östlichen Nachbarn zu nutzen. ${ }^{8}$ Dieses Ziel fand in einer differenzierten Kulturpolitik ihren Ausdruck, die Kontrolle und Entnazifizierung mit einer doppelten Bedeutung versah und damit einem Rückfall der Deutschen vorbeugen wollte, wie Corine Defrance anmerkt: "strictu sensu beschreibt Entnazifizierung den repressiven Prozeß der Säuberung oder Épuration, gleichzeitig weist sie auf den konstruktiven Prozeß der Umerziehung hin «. ${ }^{9}$ Mit Blick auf die deutsche Jugend kamen aus der Sektion Jeunesse et Sports der französischen Militärregierung, dem späteren Service des Rencontres internationales, schon 1945 Initiativen und finanzielle Mittel für Begegnungsprogramme mit einer bewußt pädagogischen Mission, wie Alfred Grosser berichtete:

Diese Sektion erlaubte ihre materielle Verwirklichung, sie hat ihnen Leben eingehaucht, sie hat privaten Institutionen erlaubt, sich zu entfalten, sie hat zahlreichen französischen Verantwortlichen die Bedeutung einer deutsch-französischen Begegnungsarbeit verständlich gemacht. ${ }^{10}$

In einer Zeit, als es noch keine deutsch-französischen Kulturbeziehungen, sondern einzig eine französische Kulturpolitik in Deutschland gab, lag ein weiterer Schwerpunkt der französischen Besatzungspolitik in der eigenen Zo-

Vgl. allgemein zur französischen Kulturpolitik in Deutschland: Corine DEFRANCE, La politique culturelle de la France sur la rive gauche du Rhin 1945-1955, Straßburg 1994; Stefan ZAUNER, Erziehung und Kulturmission. Frankreichs Bildungspolitik in Deutschland 1945-1949, München 1994.

Corine DEFRANCE, Entnazifizienung an westdeutschen Universitäten in der Besatzungszeit, in: Kurt HocHSTUHL ( $\mathrm{Hg}$.), Deutsche und Franzosen im zusammenwachsenden Europa 1945-2000, Stuttgart 2003 (Werkhefte der Staatlichen Archivverwaltung BadenWürttemberg, Serie A Landesarchivdirektion, Heft 18), S. 43-59.

${ }^{10}$ Alfred Grosser, L'Allemagne de l'Occident 1945-1952, préface d'Edmond VerMEIL, Paris ${ }^{8} 1953$, S. 295. 
ne auf der Reorganisation von Bildung, Kultur und Forschung. Dies läßt sich an der Neugründung von Orten des institutionalisierten wissenschaftlichen Austausches (Dolmetscherinstitut Germersheim, Verwaltungshochschule Speyer, Institut für Europäische Geschichte, Akademie der Wissenschaften und der Literatur und der Universität in Mainz ${ }^{11}$ ) ablesen, die damals zu den Spezifika der französischen Kulturpolitik gehörten und heute etablierter Bestandteil der deutschen Wissenschaftslandschaft sind. In der Nachkriegszeit waren sie als Instrumente der rééducation konzipiert und zielten auf die Ausbildung neuer Eliten ab, um den Prozeß der Demilitarisierung, Demokratisierung und »Déprussification « gesellschaftlich und kulturell abzusichem. Nach der Gründung der Bundesrepublik dehnte Frankreich unter Hochkommissar André François-Poncet seine Kulturpolitik über die Grenzen der ehemaligen Besatzungszone aus und baute ein Netz von Instituts Français und ähnlichen offiziellen Einrichtungen auf, ${ }^{12}$ die es in die Position versetzen sollten, mit den anderen westlichen Siegermächten im kulturellen Bereich zu konkurrieren. ${ }^{13}$

\section{Aufbau und Entwicklung eines zivilgesellschaftlichen Kommunikationsnetzes in der Bundesrepublik}

Zu den Charakteristika der französischen Kulturpolitik in den Nachkriegsjahren gehörte der Einsatz einer hohen Anzahl von Vertretern aus der Zivilgesellschaft und hier besonders aus dem Bildungsbereich (Raymond Schmittlein, Jean Moreau, César Santelli u.a.), ${ }^{14}$ die in diesen Jahren zwischen Besatzungs-

"Vgl. zu weiteren Literaturangaben jetzt: Corine DEFRANCE, Mainz in der französischen Kulturpolitik, 1945-1951, in: Mainzer Zeitschrift. Mittelrheinisches Jahrbuch für Archäologie, Kunst und Geschichte, 98 (2003), S. 73-84.

$12 \mathrm{Vgl}$. Les rapports mensuels d'André François-Poncet, Haut-Commissaire français en Allemagne 1949-1955, hg. von Hans Manfred Bock, 2 Bde., Paris 1996; Corine DEFRANCE, La création du réseau de centres culturels français en Allemagne dans l'immédiat après-guerre, in: Lendemains, 26 (2001) 103/104, S. 83-96; DIES., La Fondation de l'Institut Français et la mise en place du réseau de centres culturels français en Allemagne dans l'immédiat après-guerre, in: Nathalie PRAT, Jean-Pierre OSTERTAG, Institut Français de Mayence 1951 bis 2001. 50 Jahre französische Kultur in Mainz. "... Mayence ist das Tor zu Frankreich, das Tor zu Deutschland... «, Mainz 2001, S. 15-38.

13 Vgl. Corine DEFRANCE, Les relations universitaires franco-allemandes avant 1963. Impulsions institutionnelles et initiatives privées, in: Lendemains, 27 (2002) 107/108, S. 202-219.

14 Innerhalb der französischen Administration arbeiteten Germanisten, deren Deutschlandbilder maßgeblich auf die französische Deutschlandpolitik einwirkten; vgl. Corine DEFRANCE, Le rôle des germanistes dans la politique universitaire de la France en Allemagne pendant la période d'occupation (1945-1949), in: Lendemains, 26 (2001) 103/104, S. 56-67. 
verwaltung, Ministerialbürokratie und Bildungseinrichtungen wechselten. Diese personelle Durchlässigkeit erlaubte eine schnelle Verbreitung des Verständigungsimpulses der ersten Nachkriegsjahre, den die Protagonisten der bilateralen Begegnung und Kommunikation in die geduldige Kleinarbeit der institutionellen und privaten Austauschaktivitäten zwischen beiden Ländern in den 1950er Jahren einbrachten. Zu ihnen gehörte der gebürtige Münchner Joseph Rovan (1918-2004), der 1933 nach Frankreich emigriert und im Juli 1944 nach Dachau deportiert worden war. Er kam am 1. Juni 1945 nach Paris zurück und veröffentlichte im Oktober 1945 in der Zeitschrift Esprit den Artikel "L'Allemagne de nos mérites", in dem er von einem moralischdemokratischen und universalistischen Ansatz ausgehend die französischen Besatzungspolitiker mahnte, "daß Deutschland nicht unter Ungerechtigkeit und Unordnung derselben Natur zu leiden hat wie die, welche Deutschland Frankreich zugefügt hatte [...]. Wir können die Menschenrechte nicht durch einen Gegenrassismus mit umgekehrten Vorzeichen korrigieren, der uns ein Recht auf Rache, auf Blutrache, geben würde $\lll$. Er forderte, die Deutschen für die Sache der Demokratie zu gewinnen und wies auf die große Verantwortung der Alliierten hin: ${ }^{15} "$ Das Deutschland von morgen wird das $\mathrm{MaB}$ unserer Verdienste sein ${ }^{16}{ }^{16}$ Als Vertreter der Erwachsenenbildungsinstitution "Peuple et Culture« stieß er zur Kulturabteilung der französischen Militärregierung bzw. des Hochkommissariats (1947-1951) und kehrte 1954 in die gesellschaftliche Erziehungsarbeit zurück, um eine publizistische Karriere einzuschlagen. Später spielte er eine entscheidende Rolle bei einem der wichtigsten Schrittmacher des sozio-kulturellen Austausches, dem "Bureau International de Liaison et de Documentation « (BILD). Dies war vom Jesuitenpater Jean du Rivau (1903-1970) ${ }^{17} 1945$ in Offenburg ins Leben gerufen worden war und ging mit der "Gesellschaft für übernationale Zusammenarbeit" (GÜZ) eine Zwillingspartnerschaft ein. Beide gründeten noch im August die Zeitschriften "Documents" und "Dokumente«, die als Kommunikationskanäle über den Rhein hinweg konzipiert waren. ${ }^{18}$

Vgl. Joseph RovaN, Contes de Dachau, Paris 1987; Ders., Mémoires d'un Français qui se souvient d'avoir été Allemand, Paris 1999, S. $234 f$.

16 Vgl. Ders., Zwei Völker - eine Zukunft. Deutsche und Franzosen an der Schwelle des 21. Jahrhunderts, München 1986, S. 83-102.

17 Er hatte ab 1940 im Widerstand gegen die Nationalsozialisten gekämpft und war nach seiner Festnahme 1944 nach Mauthausen und später nach Dachau deportiert worden; vgl. Michel GURVEL, Le fondateur Jean du Rivau, in: Documents, 45 (1990) 1, S. 125-131.

$18 \mathrm{Vgl}$. Rä̈ssa MÉZIĖRES, Documents, revue des questions allemandes et l'idée européenne, 1945-1949, in: Bulletin de l'Institut Pierre Renouvin (Université de Paris I-PanthéonSorbonne), 5 (1998), S. 33-50; Henri MÉNUDIER, La revue Documents et le BLLD, in: Passerelles et Passeurs (Hommages à Gilbert Krebs et Hansgerd Schulte), Asnières 2002, S. 233-256. 
Rovan fand in Alfred Grosser (geb. 1925 in Frankfurt) einen Mitstreiter, der sich für die deutsch-französische Aussöhnung als Generalsekretär im »Comité français d'échanges avec l'Allemagne nouvelle« engagierte, das 1948 auf Initiative von Emmanuel Mounier (1905-1950), dem Gründer der Zeitschrift Esprit, ins Leben gerufen worden war. ${ }^{19}$ Das sogenannte "Grosser-Comité" wollte - unabhängig von der französischen Besatzungsmacht in Deutschland und ihren offiziellen Kulturinstitutionen - den Dialog mit der deutschen Gesellschaft auf privater Ebene aufnehmen und gemeinsam mit seinem deutschen Partner, dem im Februar 1949 unter maßgeblicher Einwirkung von Carlo Schmid eröffneten Deutsch-Französischen Institut in Ludwigsburg (DFI), ${ }^{20}$ zum demokratischen Neuanfang in Deutschland beitragen. Das Engagement seiner Mitglieder für die deutsch-französischen Beziehungen entstand zumeist aus dem Geist ihres Engagements in der Résistance ${ }^{21}$ und dem Willen, den Trägern des Demokratisierungsprozesses in Westdeutschland die Hand zu reichen, damit dort nicht wieder die Fürsprecher nationalistischer Ideen die Oberhand gewannen. Mit den Begegnungsveranstaltungen und Podiumsdiskussionen bot das Comité im kleinen Maßstab einen vorpolitischen Raum bzw. eine transnationale Öffentlichkeit, »in der Deutungsangebote bezüglich der deutschen und französischen Gesellschaft bzw. ihren Beziehungen zueinander von Intellektuellen artikuliert und gehört werden konnten . $^{22}$ Die Zielgruppen des Comité wie auch der oben angeführten Organisationen ging jedoch über den Kreis der kulturellen Elite hinaus, wie Alfred Grosser 1953 in bewußter Abkehr von den gesellschaftlichen Beziehungen zwischen Deutschland und Frankreich in der Zwischenkriegszeit betonte:

Die Definition des Wortes »Kultur« muß hingegen bedeutend erweitert werden. Die zeitgenössische französische Kultur, die für Deutschland nützlich sein kann, ist nicht alleine Theater und Literatur, sondern auch die Forschung zu Wirtschaft und Humanismus, die verschiedenen Formen von Jugendarbeit, die Entwicklung der Wahlgeographie und der Soziologie der politischen Parteien sowie die pädagogische Entwicklung der »classes nouvelles «. ${ }^{23}$

Mit diesem gewandelten Kulturbegriff, auf den noch zurückzukommen sein wird, entwickelten die genannten Vereinigungen eine öffentlichkeits- und multiplikatorenwirksame Verständigungsstrategie sowie neue zivilgesellschaftliche Kommunikations- und Kontaktstrukturen. Ein Beispiel dafür ist die

19 Vgl. Alfred Grosser, Une vie de Français. Mémoires, Paris 1997.

$20 \mathrm{Vgl}$. Hans Manfred BoCK (Hg.), Projekt deutsch-französische Verständigung. Die Rolle der Zivilgesellschaft am Beispiel des DFI in Ludwigsburg, Opladen 1998.

21 Vgl. GrosSER (Anm. 10), S. 299.

22 Carla ALBRECHT, Das Comité français d'Échanges avec l'Allemagne nouvelle als Wegbereiter des Deutsch-Französischen Jugendwerkes, in: Lendemains, 27 (2002) 107/108, S. 177-189, hier S. 181.

${ }^{23}$ Grosser (Anm. 10), S. 297. 
am 24. Oktober 1949 gegründete "Deutschen Auslandsgesellschaft« Lübeck, die sich in den 1950er Jahren als Teil der neuen gesellschaftlichen Austauschstrukturen in der Bundesrepublik profilierte und von der gesellschaftlichen Basis her das Beziehungs- und Kommunikationsgeflecht mit den Nachbarländern verdichten half. Ihr Name war Programm, denn sie wollte sowohl im Ausland Interesse fur Nachkriegsdeutschland als auch in Deutschland eine neue Art von Interesse für das Ausland wecken:

Man möge nicht vergessen, daß es schwer war, Kontakte wieder herzustellen, die durch die Brutalität der vergangenen Zeit so endgültig zerrissen schienen, Vertrauen wieder aufzubauen, wo das Mißtrauen fast vollständig herrschte, Menschen $z u$ bewegen, zu uns zu kommen und sich der Gefahr auszusetzen, als Kollaborateure mit einem verachteten Volk zu gelten. Wir wollen nicht vergessen, daß in dieser Zeit die Hilfsbereitschaft des Auslandes eines jener beglückenden und auch unsere Absichten stärkenden Momente war, die uns die Zuversicht gaben, daß auch auf dem Gebiet des menschlichen Zusammenlebens allgemein eine Wendung eintreten müßte. ${ }^{24}$

Ganz diesem Ziel verpflichtet, veröffentlichte die Gesellschaft in ihrer Zeitschrift Ausblick (1949-1994) gerade in den ersten Jahren vor allem Reiseberichte und Literaturbesprechungen, die Ausdruck eines neuen transnationalen Bewußtseins mit interkulturellen Wirkungsabsichten waren. Initiator der Gesellschaft war der Lübecker Senator Dr. Georg Kalkbrenner, ${ }^{25}$ der einen Kreis von Persönlichkeiten des kulturellen und wirtschaftlichen Lebens in der Hansestadt um sich scharte und in der ersten Ausgabe des Ausblicks vom Dezember 1949 die Orientierung seiner Gesellschaft vorgab:

Eines der traurigen Ergebnisse der Hitlerherrschaft war die Isolierung des deutschen Volkes auf fast allen Gebieten des menschlichen Daseins. Es ist eine lebenswichtige Aufgabe, diese Isolierung zu beseitigen. In neuerer Zeit mehren sich die Anzeichen, daß im Auslande die Neigung wächst, uns bei der Lösung dieser Aufgabe entgegenzukommen. Es gilt, solche Anzeichen richtig zu werten und daraus die Folgerungen zu ziehen [...]. Die Gesellschaft soll zunächst einmal ein Zeichen der Bereitschaft und des Willens zur zwischenstaatlichen Verständigung von deutscher Seite aus sein.

$\mathrm{Zu}$ diesem Zweck organisierte die "Auslandsgesellschaft" insbesondere für die junge Generation und Multiplikatoren aus dem schulischen Bereich Veranstaltungen, die dem neuen Informationsbedarf dieser Zielgruppe entsprechen sollten. ${ }^{26}$ Mit dieser Ausrichtung auf die europäischen Integrationsbemühun-

Soweit die Zitate nicht eigens gekennzeichnet sind, stammen sie aus: Deutsche Auslandsgesellschaft, Lübeck (Hg.), Unsere Auslandsarbeit 1949-1964. Ein Bericht, Lübeck 1964.

25 Vgl. Ahasver VON BRANDT, Georg Kalkbrenner $\dagger$, in: Hansische Geschichtsblätter, 75 (1957), S. 1-3.

${ }^{26}$ Neben Ausstellungen, Musik- und Theaterveranstaltungen sowie Austauschprogrammen für Lehrer und Jugendliche wurden Geisteswissenschaftler aus den nord- und westeuropäischen Ländern sowie aus Amerika eingeladen. Über Frankreich referierten zwischen 1949 und 1964 insgesamt 34 Wissenschaftler, unter ihnen die Germanisten Maurice Bou- 
gen unter abendländischen Vorzeichen stellte sich die "Auslandsgesellschaft" in den Geist der Nachkriegszeit, der in den folgenden Jahren die Grundlage für ihre Anschlußfähigkeit an größere gesellschaftliche Milieus und Bewegungen war und sie in den Kreis der oben genannten sozio-kulturellen Mittlereinrichtungen zwischen der Bundesrepublik und Frankreich führte.

Die geographische Lage der Stadt Lübeck und ihre historische Rolle als "Königin der Hanse" machte die skandinavischen Staaten zu den natürlichen Partnern der "Auslandsgesellschaft«, doch bemühte sie sich von Beginn an genauso intensiv um Frankreich. Bereits im Jahre 1950 suchte sie mit der Teilnahme an der Tagung der Deutsch-Französischen Gesellschaften in Schluchsee den Kontakt zum deutsch-französischen Mittlermilieu und fand gleichzeitig Unterstützung bei dem von Adenauer berufenen Generalkonsul bzw. Geschäftsträger der Bundesrepublik Deutschland in Paris, Wilhelm Hausenstein (1882-1957). ${ }^{27}$ Indem die Gesellschaft 1952 eine Gruppe von Lehrern aus Schleswig-Holstein und Niedersachsen zu Hospitationen nach Frankreich vermittelte, erwarb sie sich im äußersten Norden der Bundesrepublik den Ruf eines privilegierten Partners für bilaterale Austauschprogramme und arbeitete nach 1963 eng mit dem DFJW zusammen. Früh hatte die französische Regierung die Lübecker "Auslandsgesellschaft" schätzen gelernt und übergab ihr aus Anerkennung eine umfangreiche französische Bibliothek, die sie seit den 1950er Jahren in ihren Räumen in der "Breiten Straße 48 « beherbergte. ${ }^{28}$

Kalkbrenners Grundhaltung, ${ }^{29}$ jegliche politisch-administrative oder parteipolitische Instrumentalisierung der privaten Verständigungsorganisationen zu verhindem, traf sich mit den Interessen der anderen sozial und kulturell engagierten privaten Mittlerorganisationen. So wurde in den Statuten des im September 1956 gegründeten FFreundschaftskreises Rheinland-Pfalz - Burgund «

cher (nHeimat, Vaterland und Menschheit - oder über Leben und Krankheit der Begriffe $\lll$ ), Claude David (»Literarische Beziehungen zwischen Deutschland und Frankreich und der Politologe Alfred Grosser ("Deutsch-französische Beziehungen seit 1945

${ }^{27}$ Vgl. Georg Kalkbrenner an Wilhelm Hausenstein, 1.8.1951, und die Antwort von Hausenstein, 7.8.1951; PA/AA, Nachlaß Wilhelm Hausenstein, Bd. 23, Bl. 3-5.

${ }^{28}$ Darüber hinaus war sie von 1964 bis 1977 in Norddeutschland der offizielle Ansprechpartner für den Einzelschüleraustausch mit Frankreich. Der 1977 gegründete DeutschFranzösische Arbeitskreis machte es sich bis zur Einstellung seiner Aktivitäten im Jahre 2003 zum Ziel, die Verbindungen mit dem Nachbarland zu erweitern, Einblicke zu geben in Geschichte, Kultur und Wirtschaft und die Kenntnis der Sprache zu fördern. Von 1978 bis 1989 führte er in den Sommerferien ein Schüleraustausch-Programm mit der Region Poitou-Charente durch, engagierte sich für die Intensivierung des Französischunterrichts und war am Zustandekommen der Städtepartnerschaft Lübeck - La Rochelle beteiligt.

29 Zum 25. Gründungsjubiläum der Gesellschaft hieß es in der Festbroschüre über ihn: »POlitische Absichten lagen ihm bei seinen Bemühungen fern. Er wollte den menschlichen Kontakt und wußte, daß er auf dem kulturellen Gebiet besonders notwendig ist«. 
ausdrücklich sein überparteilicher und überkonfessioneller Charakter festgelegt. ${ }^{30}$ Zur Stärkung des zivilgesellschaftlichen Momentes bemühten sich die Vereinigungen $a b$ 1954, die lokal und regional entstandenen Stützpunkte deutsch-französischen Verkehrs auf Bundesebene zusammenzufassen. ${ }^{31}$ Mit diesen integrativen Bestrebungen um eine gemeinsame Außenvertretung reagierten sie auf die gesteigerten gouvernementalen Aktivitäten und den Abschluß des Deutsch-Französischen Kulturabkommens vom 23. Oktober 1954, das bei ihnen die Sorge erweckt hatte, der bilaterale Gesellschafts- und Kulturaustausch solle nun überwiegend in staatliche Regie genommen werden. So gehörte die Lübecker »Auslandsgesellschaft« neben BILD, GÜZ, DFI, "Grosser-Komitee« u.a. zu den Gründungsmitgliedern des "Arbeitskreises der privaten Institutionen für internationale Begegnung und Bildungsarbeit« (APIIBB). Dieser wurde im März 1954 als Dachorganisation privater Mittlerorganisationen gegründet und bemühte sich in den folgenden Jahren besonders um die politisch-öffentliche Anerkennung und die finanzielle Sicherung der Arbeit ihrer Mitglieder.

\section{Die Begegnungsarbeit der kulturellen Akteure in der Praxis}

\section{Der merweiterte Kulturbegriff" avant l'heure}

Als Teilnehmer an den konstituierenden Treffen des APIIBB in Marly-le-Roi (1955) und Ludwigsburg (1957) machte sich die "Auslandsgesellschaft« zu einem Befürworter und Vertreter eines neuartigen Kulturbegriffs, der gleichsam Lernziele und Methoden der organisierten internationalen Begegnungen bestimmen und Vorurteile sowie Mißtrauen abbauen helfen sollte. Diesem Ziel verpflichtet waren auch die westdeutsch-französischen Städtepartnerschaften, die in der Kontinuität des transnationalen Verständigungswillens und

Vgl. Generalkonsulat in Lyon an AA, 10.10.1967; PA/AA, B 24, Bd. 607, Bl. 504.

31 Zu den Pionieren der dezentralen Austauscharbeit gehört auch das 1957 gegründete "Comité de Liaison Normandie - Basse-Saxe«, dessen treibende Kraft Raymond Adline, Deutschlehrer am Lycée von Deauville, war. Die Tätigkeit des "Comité« erstreckte sich in den Anfangsjahren auf die Gebiete des Jugend- und Erwachsenenaustauschs, wobei es alle sozialen Schichten ansprach. Sechsmal im Jahr brachte es eine zweisprachige Publikation mit einer Auflage von ca. 2500 Exemplaren heraus. Der Erwachsenenaustausch wurde fallweise von den Regierungsstellen in Paris, Bonn und Hannover unterstützt; die bundesdeutsche Botschaft in Paris trug einen Teil der Kosten für die Publikation, und für den Jugendaustausch erhielt das »Comité « nach 1963 Mittel vom DFJW; Bundesdeutsche Botschaft in Paris an AA, 23.6.1967; PAAA, B 24, Bd. 607, Bl. 492ff. 
der gesellschaftlich-kulturellen Initiativen zur Überwindung der wechselseitigen Ressentiments aus der Zwischenkriegszeit standen und ab 1950 - wie in der Einleitung dieses Sammelbandes bereits angedeutet - den Anfang zu einer Entwicklung von interkommunalen Verbindungen mit breitenwirksamen Dimensionen darstellten. ${ }^{32}$ Auch ihre Begegnungsprogramme waren nun nicht mehr auf eine kulturelle Elite beschränkt, sondern bezogen breite Kreise der Bevölkerung aus verschiedenen gesellschaftlichen Tätigkeitsbereichen in ihre Arbeit ein. Kennzeichnend war für den in dieser Austauschform zum Tragen kommenden »erweiterten Kulturbegriff" (ein Ausdruck, der sich erst ab Anfang der 1970er Jahre in den allgemeinen deutschen Sprachgebrauch einbürgerte, aber bereits in den 1950er Jahren der Sache nach existierte), daß weiter gefaßte Manifestationen kulturellen Schaffens in den transnationalen Austausch einbezogen wurden, während die bilateralen Treffen in den 1920er Jahren an der Hochkultur beider Länder orientiert waren. Privat organisierte Austauschbewegungen sollten weiterhin Garantie dafür sein, daß an die Stelle der nationalen "Kulturpropaganda" im jeweils anderen Land, die ein möglichst scharf umrissenes Bild der eigenen und der fremden Identität zu zeichnen sucht, ein inter- und übernationaler Erfahrungsraum eröffnet wurde, in dem mehr die Gemeinsamkeiten als die Unterschiede in den Vordergrund treten. Die Diversifizierung der sozialen Trägergruppen sollte darüber hinaus die Gelegenheit geben, möglichst zahlreiche Realitätsausschnitte des nationalen Lebens in einem Land den Partnern im anderen Land zu vermitteln.

Mit zeitlicher Verzögerung, aber nachweisbar bereits ab Anfang der 1960er Jahre fanden diese Ideen Schritt für Schritt auch Eingang in die konzeptionellen Überlegungen der bundesdeutschen Kulturpolitiker und in die Praxis der Mittlerorganisationen. So bemängelte noch im April 1964 das bundesdeutsche Generalkonsulat in Nancy in seinem Bericht an die Botschaft die fehlende Bereitschaft des Goethe-Instituts vor Ort, sich neuen gesellschaftlichen Gruppen zu öffnen: »Es werden also stereotyp dieselben Menschen angesprochen. Die erwünschte Tiefen- und Breitenwirkung bleibt damit praktisch aus.« $\mathrm{Da}$ in den anderen Goethe-Instituten in Frankreich die gleiche Erfahrung gemacht wurde, erschien es der Botschaft unumgänglich, "nach neuen Formen der Arbeit der Goethe-Institute zu suchen. Vor allem sollten breitere Kreise der französischen

Hans Manfred BocK, Europa von unten. Zu den Ursprüngen und Anfängen der deutschfranzösischen Gemeindepartnerschaften, in: Annette JÜNEMANN u.a. (Hg.), Gemeindepartnerschaften im Umbruch Europa, Frankfurt a. M. 1994, S. 13-36, hier S. 25; vgl. auch Thomas GRUNERT, Langzeitwirkungen von Städtepartnerschaften, Kehl, Straßburg 1981; Corine DEFRANCE, Les premiers jumelages franco-allemands, 1950-1963, in: Lendemains, 21 (1996) 84, S. 83-95; Ulrich PFEIL, Rendsburg - Vierzon - Bitterfeld: Ein Fallbeispiel deutsch-französischer Städtepartnerschaften im Kalten Krieg, in: Zeitschrift für Schleswig-Holsteinische Geschichte, 129 (2004), S. 141-161. 
Bevölkerung und alle sozialen Schichten angesprochen werden. ${ }^{33}$ Wie eng kulturelle Öffnung und soziale Reichweite zusammenhingen, hatte der neue Direktor des im Oktober 1965 in der "Avenue de Iéna« eröffneten Pariser Goethe-Instituts bereits verstanden, wie aus seinem Halbjahresbericht (1.1. bis 30.6.1966) hervorgeht, den die bundesdeutsche Botschaft in ihrem kulturpolitischen Jahresbericht für 1966 zitierte:

Man muß sich darüber im kJaren sein, daß unsere Aufgabe in einem Lande wie Frankreich und im besonderen in einer Stadt wie Paris nicht etwa "Volksbildungsarbeit« oder gar sogenannte "Öffentlichkeitsarbeitu sein kann, sondern $\mathrm{daB}$ es sich hier in erster Linie um eine "Offenheitsarbeit « handeln muß: Es geht darum, möglichst verschiedenartige französische Kreise mit dem heutigen Deutschland, seinen Leistungen und seinen Problemen bekanntzumachen, Interesse und Verständnis für Deutschland in seiner augenblicklichen Situation zu erwecken, ich möchte fast sagen, "den Deutschen « wieder glaubwürdig zu machen. ${ }^{34}$

Eckhard Michels bezeichnet es in diesem Zusammenhang als Glücksfall, daß sich die Eröffnung des Pariser Goethe-Instituts so lange hinausgezögert habe, denn mit der Übergabe der Verantwortung für die Programmgestaltung vom Auswärtigen Amt an die Leiter der örtlichen Goethe-Institute am 23. März 1961 und der nun nicht mehr geltenden Weisungsbefugnis durch die diplomatischen Vertretungen seien durch diesen neuen inhaltlichen Freiraum Programme möglich gewesen, die den »erweiterten Kulturbegriff" bereits in die Praxis umgesetzt hätten, noch bevor dieser während der sozial-liberalen Koalition in die öffentliche Diskussion eingeführt worden sei. ${ }^{35}$

Die oben zitierte Klage diplomatischer Vertreter in Frankreich belegt jedoch die These, daß sich die kulturellen Neuerungstendenzen nicht nur auf die Mittlerorganisationen beschränkten, sondern auch vor der bundesdeutschen Diplomatie nicht Halt machten. So geht aus dem kulturpolitischen Jahresbericht für 1967 hervor, welcher Wertschätzung sich die Goethe-Institute in Frankreich mittlerweile bei der Botschaft erfreuten:

Dabei enthalten sich die Institute bewußt jeglicher Polemik, ziehen Diskussionsredner verschiedener geistiger und politischer Provenienz hinzu und gewinnen mit dieser Methode langsam aber sicher das Vertrauen von Kreisen, die bislang der Bundesrepublik ablehnend gegenüber standen. Die Leistung der Goethe-Institute, gewisse, sich aus der geistigen und geschichtlichen Situation des Gastlandes ergebende "Tabus« gebrochen und das dem Offiziellen oft anhaftende Odium überwunden zu haben, ist nicht hoch genug zu bewerten. ${ }^{36}$

Beide Zitate in: Kulturpolitischer Jahresbericht der bundesdeutschen Botschaft in Paris für 1965, 10.6.1966; PA/AA, B 97, Bd. 376, S. 4f.

${ }^{34}$ Kulturpolitischer Jahresbericht der bundesdeutschen Botschaft in Paris für 1966, März 1967; PA/AA, B 97, Bd. 376, S. 14.

$35 \mathrm{Vgl}$. Eckhard MiCHELS, Vom Glück der verspäteten Arbeitsaufnahme: Die Anfänge des Goethe-Instituts in Paris, in: Lendemains, 26 (2001) 103/104, S. 97-107.

${ }^{36}$ Kulturpolitischer Jahresbericht der bundesdeutschen Botschaft in Paris für 1967, 6.3.1968; PA/AA, B 97, Bd. 376, S. 9. 


\section{Die Institutionalisierung der Kulturbeziehungen: eine originelle Synthese zwischen staatlicher Kulturpolitik und zivilgesellschaftlichen Akteuren}

Es waren die privaten Verständigungsorganisationen und Austauschdienste in der Bundesrepublik und Frankreich, die zwischen 1945 und 1963 eine "Luftveränderung « in den beiden Gesellschaften bewirkt hatten. Sie bewog die Regierungen beider Länder, im Élysée-Vertrag die Gründung des DFJW vorzusehen, das mit der Verabschiedung des Abkommens vom 5. Juli 1963 seine Arbeit aufnahm und sich als binationaler Akteur im Spannungsfeld zwischen politischer Administration und gesellschaftlicher transnationaler Austauschund Verständigungsarbeit etablierte. ${ }^{37}$ Das hier skizzierte zivilgesellschaftliche Beziehungsnetz und die von ihm ausgegangenen Impulse zur bilateralen Begegnung und Zusammenarbeit verlängerten sich nun in die Institutionsgeschichte des DFJW und brachten eine in allen wesentlichen Aspekten eingeübte Praxis der Begegnung und der punktuellen Zusammenarbeit zwischen Deutschen und Franzosen ein, die durch den neuen institutionellen Rahmen eine Chance der Verbreiterung und Vertiefung dieser zivilgesellschaftlichen Kooperation erhielt.

Die Zusammenführung der älteren verständigungspolitischen Akteure mit den neu auf den Plan tretenden Trägern deutsch-französischer Begegnungsaktivitäten unter dem institutionellen Dach des DFJW vollzog sich in mehreren Schritten und Formen. Die in der Bundesrepublik und in Frankreich für die Arbeit des DFJW zuständigen Ministerien (»ministères de tutelle«) - das Familien- und Jugendministerium auf der einen, das Jugend- und Sportministerium auf der anderen Seite - hielten im Rahmen dieser institutionellen Gegebenheiten zwar die Macht zur Besetzung der wichtigsten Führungsgremien (Generalsekretariat, Kuratorium des DFJW) in den Händen; sie konnten sich jedoch in der Ausübung dieser Macht auf ein vorhandenes Ensemble von verständigungs- und jugendpolitischen Organisationen stützen und sich in der Ausgestaltung der Begegnungsprogramme auf deren Kompetenz und Kreativität verlassen. Auf dieser institutionellen Grundlage vollzog sich ab 1963 der größte Teil der sozio-kulturellen Austauschvorgänge zwischen der Bundesrepublik Deutschland und Frankreich. In diesem institutionellen Handlungsrahmen und durch die dort wirkenden Akteure wurde die Leitidee des werweiterten Kulturbegriffs « in immer neuen Anläufen, die von der gesellschaftlichen Entwicklung beider Nationen diktiert wurden, in die Praxis umgesetzt. Die Darstellung einiger Entwicklungslinien in dieser Kooperations- und Förde-

37 Vgl. zu seiner Gründung und Arbeit jetzt: Hans Manfred Bock (Hg.), Deutsch-französische Begegnung und europäischer Bürgersinn. Studien zum Deutsch-Franzősischen Jugendwerk 1963-2003, Opladen 2003. 
rungspraxis, die zumeist experimentell verlief, mag die Entstehung und Eigenart des Modells deutsch-französischer Kulturbeziehungen seit 1963 genauer hervortreten lassen, dessen Prinzipien in den nachfolgenden vierzig Jahren nicht wirklich mehr in Frage gestellt wurden. ${ }^{38}$

Während der ersten beiden Jahrzehnte der Arbeit des DFJW fügten sich die drei (ministeriellen, institutionellen und organisationellen) Bauelemente in einem längeren Vorgang des Um- und Anbauens zu einer funktionsfähigen Einheit zusammen, die in ihrem Grundriß beständiger blieb, als man es nach den eher schwierigen Anfängen der neuartigen binationalen Kulturinstitution hätte erwarten können. ${ }^{39}$ Die Dauerhaftigkeit dieser funktionalen Synthese ist ein Indiz dafür, da $B$ alle beteiligten Akteure aus ihr für die Erfüllung ihrer spezifischen Zweckbestimmung Gewinn zogen. Die für die Jugend zuständigen Ministerien beider Länder erhielten im Rahmen dieser Synthese zusätzliche Gestaltungsmöglichkeiten und im jugendverbandlichen Bereich eine Infrastruktur für die materielle Umsetzung des politischen Ziels der Verbesserung der deutsch-französischen Beziehungen. Das DFJW erhielt von der öffentlichen Hand die finanziellen Ressourcen und aus dem Bereich der Jugendverbände sowie der bilateralen Verständigungsvereine das personelle Rekrutierungsangebot und die sachliche Kompetenz. Die jugend- und verständigungspolitischen Organisationen in beiden Ländern selbst gewannen im bilateralen Handlungsbereich finanzielle Unterstützung durch den Staat und Reflexionsbzw. Kontaktherstellungschancen durch die zentrale Institution DFJW.

Im Interaktionsfeld der gesellschaftlichen Verbände bedeuteten ÉlyséeVertrag und DFJW längerfristig eine Stabilisierung der bilateralen Austauschorganisationen und eine Vervielfachung der im deutsch-französischen Begegnungsverkehr tätigen Organisationen, die ihre internationalen Kontakte ab 1963 um einen deutsch-französischen Schwerpunkt ergänzten. Die älteren privaten Mittlerorganisationen, deren Handschrift besonders die frühesten Richtlinien für die Förderungspraxis des DFJW trugen, waren in dem sich alle zwei Jahre erneuernden Kuratorium des DFJW quantitativ zwar eher schwach vertreten, spielten aber vor allem in der Person hervorragender Sprecher (u.a. Joseph Rovan und Alfred Grosser) in dessen Sitzungen eine reflexive Schrittmacherrolle. Gerade in der Frühgeschichte des DFJW stellten sie jene selbstkritischen Kräfte, die um die pädagogisch und technisch angemessene Planung, Durchführung und Evaluierung der konkreten Austauschmaßnahmen zwischen

Teilweise erfolgt eine solche Infragestellung gegenwärtig durch eine deutsch-französische Parlamentarier-Arbeitsgruppe; vgl. deren Ausarbeitung: Bericht der Arbeitsgruppe des Deutschen Bundestages und der Assemblée nationale zum Deutsch-Französischen Jugendwerk, 23. Juni 2004.

39 Vgl. dazu die einschlägigen ersten vier Kapitel in BocK (Anm. 37), S. 13-122. 
jungen Deutschen und Franzosen Sorge trugen. Diese Sorge brachte Alfred Grosser Anfang der 1970er Jahre auf die griffige Formel »Massenwandern statt Nachdenken? «. $^{40}$ Gemeint war damit die Gefahr, daß gerade bei den zahlreich als Antragsteller für die Förderung von Austauschmaßnahmen neu auf den Plan tretenden Verbänden infolge ihrer mangelnden interkulturellen Kompetenz mehr Schaden als Nutzen für die wechselseitige Kenntnis deutscher und französischer Jugendlicher gestiftet wurde. Höhepunkt der entsprechenden internen Kritik war die Tagung aus Anlaß des fünfjährigen Bestehens des DFJW im Jahre $1968 .{ }^{41}$ Es scheint, daß die Reformen des DFJW, die in den Jahren 1974/75 eingeleitet wurden, die Ursachen für diese Kritik teilweise beseitigten; das Problem eines effektiveren und verbesserten transnationalen und interkulturellen Lernens voneinander und miteinander blieb aber ein zentrales Thema, dem sich das DFJW in den 1980er Jahren auf neuen Wegen näherte. ${ }^{42}$ Diese schwierig zu lösende Aufgabe war in den Prämissen des »erweiterten Kulturbegriffs« und in der Erweiterung der gesellschaftlichen Trägergruppen für den transnationalen Austausch angelegt. Die Tatsache, daß die Dachverbände der privaten Austauschdienste, die in Frankreich Ende der 1940er Jahre und in der Bundesrepublik Mitte der 1950er Jahre gegründet worden waren, in den frühen 1970er Jahren ihre Tätigkeit einstellten ${ }^{43}$ kann als Hinweis dafür interpretiert werden, daß ihre Zielsetzungen in der DFJW-Synthese aufgehoben zu sein schienen.

Die jugendpolitischen Organisationen, die mit dem "Deutschen Bundesjugendring" (DBJR) und dem "Conseil français des mouvements de jeunesse» über eigene Dachverbände verfügten, waren seit 1963 überwiegend Neuankömmlinge im bilateralen deutsch-französischen Gespräch und wurden anfangs von den älteren Protagonisten dieses Dialogs skeptisch beäugt. Sie hatten den Vorkämpfern der deutsch-französischen Verständigung voraus, daß sie in einem eingeübten Verhältnis zu den Jugendministerien standen, die für die Ingangsetzung der neuen DFJW-Strukturen verantwortlich waren. Aus diesem Sachverhalt erklärt sich die privilegierte Repräsentanz dieser Verbände des Jugendsektors in der Gruppe der nichtgouvernementalen Mitglieder in den

Vgl. Alfred Grosser, Versagen die Mittler? Was Deutschland und Frankreich voneinander wissen, in: Robert PICHT u.a. (Hg.), Perspektiven der Frankreichkunde. Ansätze zu einer interdisziplinär orientierten Romanistik, Tübingen 1974, S. 3-9, hier S. 10.

41 Vgl. Kongreß DFJW, Colloque OFAJ 1968, Paris o. J. (1969).

${ }^{42}$ Dazu Katja MARMETSCHKE, Krise und Neugestaltung des DFJW in den siebziger Jahren, in: BOCK (Anm. 37), S. 114-116.

43 Das "Comité de coordination des associations d'échanges internationauxu löste sich zu Beginn der siebziger Jahre auf und fusionierte teilweise mit einem neuen Dachverband; der Arbeitskreis der privaten Institutionen existierte bis 2002, verringerte jedoch seine nach innen und außen gerichteten Aktivitäten seit Beginn der siebziger Jahre deutlich. 
Kuratorien des DFJW. ${ }^{44}$ Besonders für diese Verbände- und Vereinskategorie wurden schon in der Anfangsphase des DFJW Finanzmittel zur Verfügung gestellt, die zur Ergänzung ihrer administrativen Kapazität um eine deutschfranzösische Komponente (in der Regel eine halbe Sekretariatsstelle) dienen sollten. Diese Anschubfinanzierung wurde nicht immer angemessen verwendet, und seit der Reform des DFJW in den frühen 1970er Jahren wurde ein weniger eng gewebtes Netz von pädagogischen Mitarbeitern eingeführt, die bei den gesellschaftlichen Organisationen arbeiten und der Pflicht zum regelmäßigen Erfahrungsaustausch und zur Rechenschaftslegung unterworfen sind.

Als funktionales Problem erwies es sich schon im ersten Jahrzehnt der binationalen Austauschinstitution, daß sich aus den Hunderten von Vereinigungen in beiden Ländern, die Projekte mit dem DFJW durchführten, ein sehr beständiger Kern von verständigungs- und jugendpolitischen Organisationen herausbildete, der zeitweilig zwei Drittel der zur Verfügung stehenden Mittel des Zweck- bzw. Interventionshaushalts für sich in Anspruch nahm. Da nur eine begrenzte Zahl der gesellschaftlichen Partnerorganisationen des DFJW die Kapazität und die Kompetenz für die Planung, Durchfuhrung sowie Auswertung bilateraler Austausch- und Begegnungsprogramme hatte und hat, ist dieses Problem nicht wirklich zu lösen, sonderm allenfalls mittels aktiver Wachsamkeit gegen Kartellbildungen und "Erbhöfe« in diesem Bereich zu begrenzen. Eine Stichprobe vom Anfang und vom Ende der 1990er Jahre zeigt, daß von den zehn größten Empfängern von DFJW-Fördermitteln für Begegnungsprogramme die Mehrheit in Deutschland wie in Frankreich solche Organisationen waren, die schon seit den 1960er Jahren zu den aktivsten Kooperationspartnern der binationalen Institution gehörten. ${ }^{45}$

Indem die deutschen oder den französischen Amtsinhaber (bzw. deren Mitarbeiter) abwechselnd die Kuratoriumssitzungen leiteten, waren die für das DFJW zuständigen Jugendministerien in der institutionellen Synthese dieser Einrichtung direkt vertreten; indirekt schalteten sie sich durch die Emennung der jeweils 10 (später 15) Kuratoriumsmitglieder und ihrer Stellvertreter sowie des Generalsekretärs und seines Stellvertreters ein. Im Verhältnis des deut-

Gemäß Artikel 6 des Abkommens vom 5.7.1963 setzten sich die jeweils zehn Mitglieder aus vier Vertretern der öffentlichen Verwaltung und aus sechs mnamhaften Persönlichkeiten und Leitern von Organisationen der freien Jugendarbeit« zusammen. Diese Relation wurde bei den Abänderungen des Abkommens von 1973 und 1983 gewahrt (ab 1983 im Verhältnis sechs zu neun); vgl. die Vertragstexte in Adolf KIMMEL, Pieme JARDIN (Hg.), Die deutsch-französischen Beziehungen seit 1963. Eine Dokumentation, Opladen 2002, S. $485,489,492$.

45 Verzeichnis der 50 wichtigsten Verbands-Partner des DFJW in der Anlage zum Orientierungsbericht 1991 (67. Kuratoriumssitzung) und zum Orientierungsbericht 2000 (85. Kuratoriumssitzung), beide im Archiv des DFJW-Kuratoriums in Paris. 
schen und des französischen Ministeriums zu den Organen des DFJW (Kuratorium und Generalsekretär) spiegeln sich unterschiedliche Traditionen der öffentlichen Verwaltungsstruktur in der Weise wider, daß die Verbindung und Interaktion der DFJW-Organe mit den Ministerialbeamten auf der deutschen Seite eher locker, auf der französischen Seite eher stark ist. Das läßt sich z.B. an der Art der Vorbereitung der Kuratoriumssitzungen beobachten, die in Frankreich in der Regel in enger Abstimmung zwischen beiden Akteursgruppen verläuft, in Deutschland hingegen in zwangloser Form. In der alle fünf Jahre fälligen Besetzung der beiden Generalsekretariatsstellen, die abwechselnd von einem Deutschen und einem Franzosen eingenommen werden und deren Stellvertreter aus der jeweils anderen Nation stammen muB, ist anhand der längerfristigen Entwicklung der Institution in beiden Ländern die Regel erkennbar, daß nach einem Regierungswechsel die Neubesetzung dieses Amtes entsprechend der politischen Parteizugehörigkeit des Jugendministers vorgenommen wird. Da gemäß dem juristischen Status des DFJW, der sich an die Stellung der Sonderorganisationen der UNO anlehnt, ${ }^{46}$ der Generalsekretär gegenüber den Jugendministerien nicht weisungsgebunden ist und entsprechend autonom die Institution leitet, spielt die gemeinsame politische Grundüberzeugung eine wichtige Rolle als Klammer zwischen der ministeriellen Ebene und der institutionellen Leitungsebene des Jugendwerks. So ist es auch nicht verwunderlich, wenn man in der Analyse der Herkunft der bisher neun Generalsekretär-Tandems des DFJW eine sehr starke Prägung derselben durch ihre vorhergehende Tätigkeit in der Ministerialbürokratie (Außen- und Jugendministerium, in Frankreich auch Erziehungsministerium) findet. In der praktischen Arbeit der binationalen Austauschinstitution ist allem Anschein nach die parteipolitische Zugehörigkeit der Generalsekretäre weit weniger ausschlaggebend als ihre persönliche Führungs- und Initiativkraft.

Die institutionelle Eigendynamik des DFJW, die im Generalsekretariat und im Kuratorium ihre Antriebsquellen hat, stellt die dritte Komponente der originellen Synthese dar, die diese Neuschöpfung der transnationalen Kulturpolitik kennzeichnet. Besonders in der Frühgeschichte des DFJW beteuerten nicht nur die Generalsekretäre, daß die neue Institution nicht an die Stelle der bislang von gesellschaftlichen Initiativen getragenen deutsch-französischen Austauscharbeit treten wolle, sondern dies wurde auch schon in den Vorläufigen Richtlinien für die Arbeit des Jugendwerks vom Januar 1964 festgeschrieben. Dort hieß es u.a.:

46 Zum internationalen Rechtsstatus des DFJW siehe Irmgard HEINEMANN, Le Traité franco-allemand du 22 janvier 1963 et sa mise en œuvre sons le général de Gaulle (19631969), thèse de $3^{\text {enxe }}$ cycle, Faculté de droit, Université de Nice 1977, S. 311-331. 
Das Jugendwerk wird die auf dem Gebiet der Begegnung und des Austauschs zwischen der deutschen und der französischen Jugend seit dem Jahre 1945 geleistete Arbeit fortsetzen und weiterentwickeln. [...] Das DFJW soll nur ausnahmsweise selbst Träger von Programmen und Maßnahmen sein. Das Jugendwerk hat nicht die Absicht, an die Stelle von Trägern zu treten, die schon in der Jugendarbeit tätig sind."

Die Möglichkeiten und die Versuchung des Generalsekretariats, die Initiative zu Lasten der zivilgesellschaftlichen Träger an sich zu reißen, war gerade im ersten Jahrzehnt der DFJW-Entwicklung aufgrund eines schmalen Personalhaushaltes und einer anfangs komplizierten Führungsstruktur gering. ${ }^{48}$ Überdies waren die Beziehungen zwischen dem Generalsekretariat und den Kuratorien in dieser Zeitspanne in den Grundfragen der praktischen Förderungspolitik besonders konflikthaltig, beispielsweise in der Frage des politischen oder unpolitischen Zuschnitts der Programme, die vor und nach dem Mai 1968 eine besondere Aktualität erhielt. In der weiteren Entwicklung des DFJW wurde das konfliktbeladene Verhältnis zwischen den (von den zuständigen Ministerien eingesetzten) Generalsekretariaten und den Kuratorien (in denen statutarisch stets eine Mehrheit aus zivilgesellschaftlichen Verbänden und Vereinen gegeben war) zwar abgemildert, tauchte jedoch fallweise immer wieder auf. Das Kuratorium und das Generalsekretariat als institutionelle Kernstrukturen des DFJW konzentrierten ihre Tätigkeit auf die Festlegung der Regularien (»Richtlinien«), die großen Querschnittaufgaben wie interkulturelles Lernen und Erlemen der Partnersprache, sowie auf die Schwerpunktsetzung in der Förderung von Austausch- und Begegnungsprogrammen, die von Verbänden, Vereinen und sonstigen Gruppen im deutsch-französischen Kontext durchgeführt wurden.

Die Richtlinien, für die gemäß Art. 9 des Abkommens über die Gründung des Jugendwerkes das Kuratorium zuständig war, wurden in der Frühgeschichte des DFJW mehrmals verändert; sie fixierten den Jugendbegriff auf die Altersgrenze bis zum 30. Lebensjahr, gliederten die Programmbereiche und schrieben das "Zentralstellenprinzip" auf Förderungsmittel für Antragsteller fest. Nach schwierigen Anfängen und eingestandenen Fehlstarts im Bereich des interkulturellen Lernens ${ }^{49}$ beschritt das Jugendwerk hier in der zweiten Hälfte der 1970er Jahre den Weg der Kooperation der Praktiker des Jugendaustauschs mit universitären Vertretern der Bildungssoziologie und der

47 Vorläufige Richtlinien des DFJW in: Deutsche Jugend, 1964, Heft 2, S. 89-93.

$48 \mathrm{Vgl}$. zur Funktion der beiden nationalen Direktionen in der Führungsebene unterhalb des Generalsekretariats u.a. Georg Waither HEYER, Das deutsch-französische Jugendwerk. Ziele, Möglichkeiten, Erfahrungen, Freudenstadt 1969.

$49 \mathrm{Vgl}$. den Versuch zur Synthese in Andreas THIMMEL, Pädagogik der internationalen Jugendarbeit. Geschichte, Praxis, Konzepte des Interkulturellen Lemens, Schwalbach am Taunus 2001, S. 169-199. 
Erziehungswissenschaften, der trotz anfänglicher Theorie-Praxis-Kontroversen später fortgesetzt wurde und zu einer größeren Zahl viel beachteter Studien zu den Problemen transnationalen Lernens führte. ${ }^{50}$ Im Bereich des Erlernens der Partnersprache, dem anderen zentralen Aufgabenfeld mit unmittelbarer Praxisrelevanz in der deutsch-französischen Jugendbegegnung, nahm sich das Jugendwerk vorzugsweise des Terrains des außerschulischen Fremdsprachenunterrichts an. Hier stellte sich eine dauerhafte Zusammenarbeit der Praxisvertreter mit der seit den 1970er Jahren expandierenden universitären Linguistik und Sprachlehrforschung nicht her; es entstanden aber aus dem $\mathrm{Zu}$ sammenwirken des DFJW-Sprachenreferats und einer größeren Zahl von Berufsfeldvertretern praktisch hilfreiche Sprachglossare, deren Gebrauchswert weit über den Gruppenbegegnungszweck hinausreicht. ${ }^{51}$

In der Programmgestaltung für die Förderung und Strukturierung der vielfältigen Begegnungsaktivitäten, die von den öffentlichen oder den privaten Trägerorganisationen beantragt wurden, liegt zweifellos das stärkste Steuerungsinstrument, über das die Führungsgremien des DFJW verfügen. In der langfristigen Entwicklung dieser Gestaltungsfunktion durch das Kuratorium und das Generalsekretariat sind mindestens zwei Trends zu erkennen: Eine große Beweglichkeit der Programmtypen, ein Neuauftauchen und ein Verschwinden von Begegnungskategorien im deutsch-französischen Jugendaustausch und eine kontinuierlich wachsende Zahl von Programmtypen, die nicht von den Trägerorganisationen bzw. -institutionen kreiert, sondern von den Führungsgremien des DFJW selbst definiert und lanciert wurden. An die Stelle der Programmgruppe "Gemeinsame Kinder-, Jugend-, Familienerholung und Familienaustausch « z.B., die in den Anfängen des DFJW im Rahmen des Einzelaustauschs noch eine nicht unbedeutende Rolle spielte, traten verstärkt seit den 1980er Jahren andere Programmschwerpunkte, die im Zusammenhang mit der neuartigen Situation der damaligen Jugend in beiden Ländern standen (Jugendarbeitslosigkeit, Drogensucht, AIDS-Prävention). Mit diesen neuen Programmgruppen dehnte das DFJW den »erweiterten Kulturbegriff « am weitesten aus zur gemeinsamen, gleichsam sozialpädagogischen Bearbeitung brennender kollektiver Probleme der Jugendlichen in beiden Ländern; es ging damit an die Grenze der allgemein akzeptierten Auffassung von Kultur, die durch den Siegeszug massenkultureller Phänomene allerdings selbst in Bewegung geriet. Dabei ist in der Programmgestaltungsfunktion der DFJW-Steuerungsgremien in der Langfristperspektive erkennbar, daß neue Programmcluster zunehmend aus seiner eigenen Initiative hervorgingen; dies ist eine Entwicklung, die in deutlichem Kontrast zur Praxis des ersten Jahr-

so $\mathrm{Vgl}$. dazu die Bibliographie in Bock (Anm. 37), S. $330 \mathrm{f}$.

si Vgl. ibid. S. 332f. 
zehnts steht. Offensichtlich wird dies in einigen Programmschwerpunkten, die sich seit den 1980er Jahren sehr dynamisch ausweiteten. ${ }^{52}$ Das DrittländerProgramm, das statutarisch lange Zeit auf $5 \%$ der durchgeführten Maßnahmen begrenzt blieb, wurde 1989 auch auf Nicht-EG-Länder ausgeweitet, und das DFJW übernahm ab der Wende von 1989/90 zahlreiche selbstgenerierte und durch Sonderfonds finanzierte jugendpolitische Missionen in den ost- und mitteleuropäischen Ländern.

Im Umfang nicht vergleichbar und intern durchaus umstritten ist das Förderungsprogramm für junge Künstler, das bis in die zweite Hälfte der 1970er Jahre auf Verbandsebene keine Fürsprache fand, dann aber aus der Referatsebene des DFJW heraus lanciert und erfolgreich durchgeführt wurde. Auch in diesem Tätigkeitsbereich stellte sich regelmäßig die Frage nach der Dehnbarkeit des Kulturbegriffs, und zwar in diesem Fall nach seiner Offenheit zur Hochkultur. Die feststellbare verstärkte Programmdefinition durch Eigeninitiative des DFJW steht zumindest teilweise im ursächlichen Zusammenhang mit der weithin konstatierten und beklagten Veränderung der in der Jugendwerksarbeit beteiligten Verbände und Vereine, in denen mit der Schwächung des Elements des freiwilligen und unentgeltlichen Engagements der Mitglieder die wesentliche Antriebskraft für ihre transnationale Verständigungsarbeit zu schwinden scheint.

Die Wirkung des Élysée-Vertrages und des DFJW auf die bilateralen Gesellschafts- und Kulturbeziehungen sind quantitativ unbestreitbar positiv, qualitativ in jedem Fall konstruktiv - wenngleich schwieriger zu messen. Für den erstgenannten Aspekt, die quantitative Ausweitung, sprechen die Zahlen für sich selbst. Bereits in den ersten vier Jahren wurden 1080540 Jugendliche im Rahmen von 28151 Begegnungen zwischen der Bundesrepublik Deutschland und Frankreich zusammengeführt; ${ }^{53}$ für die vorausgehende Zeitspanne von 1945 bis 1963 waren rund 200000 junge Franzosen im Rahmen organisierten Austauschs nach Deutschland gekommen, allein in den Jahren 1963 bis 1967 waren es dann schon $506917 .{ }^{54}$ In den 40 Jahren seiner Tätigkeit förderte das DFJW die Begegnung von 7,2 Millionen jungen Franzosen und Deutschen in rund 235000 Programmen. In qualitativer Hinsicht entstand in der Folge der Gründung dieser ersten binationalen Institution des Kulturaustauschs zwischen Deutschland und Frankreich im Zeichen eines werweiterten Kulturbegriffs"

Zu den Drittländerprogrammen und zu den Künstlerprogrammen, auf die im folgenden Bezug genommen wird, vgl. die einschlägigen Beiträge von Carla ALBRECHT und AnneKathrin AUEL in: BOCK (Anm. 37), S. 287-301 und S. 267-286.

54 Diese Zahlen in: Untersuchung über den zahlenmäBigen Stand und die Art des deutschfranzösischen Jugendaustauschs vor Gründung des DFJW. Aufenthalt junger Franzosen in Deutschland, ungedrucktes Ms. (13 S.) im Kuratoriums-Archiv des DFJW in Paris. 
und der Demokratisierung der Gruppenteilhabe am transnationalen Austausch eine überaus originelle Austauschpraxis, die von ministeriellen, eigeninstitutionellen und verbandlichen Akteuren gestaltet wird und in der die Selbstkritik immer einen Impuls der Emeuerung darstellte. In Zukunft werden die Parlamente beider Länder bei den Beratungen über die Neugestaltung des DFJW daher gut beraten sein, wenn sie die Originalität dieser zukunftsfähigen institutionellen Synthese zur Kenntnis nehmen und respektieren. 